\title{
Fermions and Disorder in Ising and Related Models in Two Dimensions
}

\author{
V.N. Plechko \\ Bogoliubov Laboratory of Theoretical Physics, \\ Joint Institute for Nuclear Research, 141980 Dubna, Russia
}

\begin{abstract}
The aspects of phase transitions in the two-dimensional Ising models modified by quenched and annealed site disorder are discussed in the framework of fermionic approach based on the reformulation of the problem in terms of integrals with anticommuting Grassmann variables.
\end{abstract}

\section{Introduction}

The two-dimensional (2D) Ising model (2DIM) plays important role in the theory of phase transitions and critical phenomena due to the analytic results available (in pure case) over the whole temperature range [1, 2, 3, 4. In this report, we review the new mathematical methods of analysis and the results so far obtained for 2DIM modified by site disorder with application of the anticommuting (Grassmann) integrals. The Ising model by itself, in its original formulation, is a lattice model of a ferromagnet presented by a set of Ising spins $\sigma_{m n}= \pm 1$ interacting with their nearest neighbours along the lattice bonds [1, 2, 3, 4]. The modern approaches to Ising models are merely based however on the fermionic path integral reformulation of the problem in terms of the integrals with anticommuting Grassmann variables [5, 6, 7, 8, 9, 10. The advantage of the use of Grassmann variables is that they are canonical variables, as distinct from Ising spins, so that one can pass to the momentum space for fermions [5, 6, 9, 10]. In the pure case, the fermionization of 2DIM results the Gaussian fermionic integral for the partition function, $Z$, which in essence means the exact solution of the problem [5, 6, 8, 9, The formulations of this kind also admit the interpretation of the 2D Ising model as a lattice quantum field theoretical (QFT) problem [11, 12, 13, 14. In particular, the pure 2DIM on a rectangular lattice may be presented by the Majorana action with two-component massive fermions on a lattice [14. By doubling the number of fermions, one can pass as well to the Dirac action

\footnotetext{
* The present discussion is partly based on a talk given at the International Bogoliubov Conference on Problems of Theoretical and Mathematical Physics, MIRAS-JINR, Moscow-Dubna, Russia, August $21-27,2009$.
} 
[14]. The effects of disorder in 2DIM have been extensively studied during last decades both theoretically and in the precise Monte-Carlo simulations [11]-[38]. The disordered versions of 2DIM may assume either random modification of the interaction along the lattice bonds [11, 12, 15, 17, 18, [30, 31], or an admixture of the random nonmagnetic impurities at lattice sites [14, 19, 22, 25, 26, 27, 28], also see [32, 33, 34, 35, 36]. In each of this cases one may be interested, motivated by physical considerations, and possible applications, in either quenched or annealed versions of disorder. In the quenched version, the impurities are assumed to be frozen over the sample. In this case their distribution does not depend on temperature and other tuning parameters, like magnetic field, and one have to average rather the free energy $-\beta F=\ln Z$ than the partition function $Z$ itself, over the impurities [11, 12, 13, 14]. In the annealed version, the impurities may be created and annihilated by a variation of external parameters and their concentration is governed by the temperature rate and associated chemical potentials [32, 33, 36, 38]. In this case, one has to average in $Z$ itself over all states [33, 34, 35, 36. In essence, for annealed site disorder, the dilute site can be viewed as being presented by additional (zero) component of Ising spin. The resulting model is also known as the spin-1 Ising model, or the Blume-Capel model [32, 33, 36, 38]. The basic variable in the Blume-Capel model is $S_{m n}=0, \pm 1$. The modifications introduced by disorder of any kind typically result in the appearance of the additional non-Gaussian terms in fermionic action. Despite of the non-Gaussian action in the fermionic integral for $Z$, the precise results can still be derived for disordered 2D Ising models [11, 12, 13, 14, 36]. In what follows, we only consider the generic case of the random-site disorder (site dilution) introduced by adding some amount of nonmagnetic impurities into a sample, which may be either quenched or annealed, and discuss the consequences that can be derived from the fermionic integral representations for the partition functions of that models.

\section{The quenched site dilute Ising model}

The basic variable in the pure 2DIM is the dichotomic Ising spin $\sigma_{m n}= \pm 1$. The spins are disposed at the sites of a regular two-dimensional lattice and interact with nearest neighbours along the lattice bonds. The disordered version (quenched site dilution) assumes that some sites may be nonmagnetic at random. It is suitable to introduce such sites by adding the variable $y_{m n}=0,1$ at each $m n$ site, corresponding to the magnetic moment of Ising spin at a given site [14]. The resulting hamiltonian is:

$$
H\{y \mid \sigma\}=-\sum_{m n}\left[J_{1} y_{m n} y_{m+1 n} \sigma_{m n} \sigma_{m+1 n}+J_{2} y_{m n} y_{m n+1} \sigma_{m n} \sigma_{m n+1}\right]
$$

where $J_{1,2}$ are the ferromagnetic exchange energies; the lattice sites are marked by discrete coordinates $m n$, where $m, n=1,2, \ldots, L$, are running in horizontal and vertical directions, respectively; we put $L^{2} \rightarrow \infty$ at final stages. For fixed disorder, the partition function and free energy are defined by the canonical equations: $Z\{y\}=\Sigma \exp (-\beta H\{y \mid \sigma\})=$ $\exp (-\beta F\{y\})$, where the sum is taken over the all possible spin configurations provided by $\sigma_{m n}= \pm 1$ at each site. The hamiltonian modulus in the Gibbs exponential is: 


$$
-\beta H\{y \mid \sigma\}=\sum_{m n}\left[b_{1} y_{m n} y_{m+1 n} \sigma_{m n} \sigma_{m+1 n}+b_{2} y_{m n} y_{m n+1} \sigma_{m n} \sigma_{m n+1}\right],
$$

where $b_{1,2}=\beta J_{1,2}$, and $\beta=1 / k T$ is the inverse temperature. For a typical bond weight from $Z$ we write: $\exp \left(b y y^{\prime} \sigma \sigma^{\prime}\right)=\cosh \left(b y y^{\prime}\right)+y y^{\prime} \sigma \sigma^{\prime} \sinh (b)$, since $\sigma \sigma^{\prime}= \pm 1$ and $y y^{\prime}=0,1$. The partition function can then be written in the form: $Z\{y\}=$ $R\{y\} Q\{y\}$, where $R\{y\}$ is a nonsingular spin-independent prefactor, formed by a product of $\cosh \left(b y y^{\prime}\right)$, while $Q\{y\}$ is the reduced partition function:

$$
Q\{y\}=\operatorname{Sp}_{(\sigma)}\left\{\prod_{m n}\left(1+t_{1} y_{m n} y_{m+1 n} \sigma_{m n} \sigma_{m+1 n}\right)\left(1+t_{2} y_{m n} y_{m n+1} \sigma_{m n} \sigma_{m n+1}\right)\right\},
$$

where $t_{1,2}=\tanh b_{1,2}$, and we assume a properly normalized spin averaging, such that $\operatorname{Sp}(1)=1$ and $\operatorname{Sp}\left(\sigma_{m n}\right)=0$ at each site. In given case, since we are interesting in quenched disorder, we have to average over $y_{m n}=0,1$ rather the free energy $-\beta \ln Z\{y\}$ than the partition function $Z\{y\}$ itself. The prefactor $R\{y\}$ provides only additive nonsingular contribution like $\ln R\{y\}$ to $\ln Z\{y\}$ and will be ignored in what follows. The problem thus reduces to the averaging of $-\beta F_{Q}=\ln Q\{y\}$ over $y_{m n}=0,1$ at each site. 1 The known device to avoid the averaging of the logarithm is the replica trick: $\left[-\beta F_{Q}\{y\}\right]=[\ln Q\{y\}]=\left[\frac{1}{N}\left(Q^{N}\{y\}-1\right)\right]_{N \rightarrow 0}$, where $[\ldots]$ stands for the average over the impurities. In this scheme, one takes $N$ identical copies of the original partition function and average $Q^{N}\{y\}$, with formal limit $N \rightarrow 0$ to be performed at final stages. The simplest distribution for the averaging over the impurities is assumed in what follows: $w\left(y_{m n}\right)=p \delta\left(1-y_{m n}\right)+(1-p) \delta\left(y_{m n}\right)$, where $p$ is the probability that any given site, chosen at random, is occupied by the normal Ising spin, while $1-p$ is the probability that the given site is dilute. The averaging of any function like $A\left(y_{m n}\right)$ then results: $\left[A\left(y_{m n}\right)\right]=p A(1)+(1-p) A(0)$.

The partition function with fixed disorder (3) can be transformed into a Gaussian fermionic integral following the method of the mirror-ordered factorization for the density matrix [8, 9, 14]. Introducing a pair of fermionic (Grassmann) variables $a_{m n}, a_{m n}^{*}$, we write for the horizontal weight:

$$
\begin{aligned}
& 1+t_{1} y_{m n} y_{m+1 n} \sigma_{m n} \sigma_{m+1 n} \\
& =\int d a_{m n}^{*} d a_{m n} \mathrm{e}^{a_{m n} a_{m n}^{*}}\left(1+a_{m n} y_{m n} \sigma_{m n}\right)\left(1+t_{1} a_{m n}^{*} y_{m+1 n} \sigma_{m+1 n}\right) .
\end{aligned}
$$

1 The situation is different for the annealed case (the Blume-Capel model), where the zero state provided by $y_{m n}=0,1$ is rather to be considered as a zero component of the BC spin $S_{m n}=0, \pm 1$ so that one have to average over the all three states of $S_{m n}=0, \pm 1$ directly in $Z$, inside of the logarithm. Respectively, the cosine factors (product $R\{y\}$ ) are now to be preserved under the averaging in $Z$. These factors in fact add new degrees of freedom for clusterization of the magnetic sites at low temperatures, and are eventually responsible for the appearance of the tricritical point in the Blume-Capel model at strong dilution [36. In fermionic language, the tricritical point is associated with vanishing of the kinetic (stiffness) coefficient in the Blume-Capel fermionic action at strong dilution [36]. 
In a conventional notation, the horizontal weight is now presented as a product of two factors, $A_{m n} A_{m+1 n}^{*}$, with decoupled spins, taken under the Gaussian averaging. 2 In a similar way, one prepares the factorized vertical weights in the form $B_{m n} B_{m n+1}^{*}$. At next stage, we have to arrange the factors in their global products in order the elimination of spin variables be possible [8, 9, 14]. The final point is that we have to average over $\sigma_{m n}= \pm 1$ the product of four factors with the same spin like $A_{m n}^{*} B_{m n}^{*} A_{m n} B_{m n}$ at each site, thus passing to a purely fermionic expression. This results the integral [14]:

$$
\begin{aligned}
& Q\{y\}=\int \prod_{m n} d b_{m n}^{*} d b_{m n} d a_{m n}^{*} d a_{m n} \exp \sum_{m n}\left\{a_{m n} a_{m n}^{*}+b_{m n} b_{m n}^{*}+\right. \\
& \left.+y_{m n}^{2}\left[a_{m n} b_{m n}+t_{1} t_{2} a_{m-1 n}^{*} b_{m n-1}^{*}+\left(t_{1} a_{m-1 n}^{*}+t_{2} b_{m n-1}^{*}\right)\left(a_{m n}+b_{m n}\right)\right]\right\},
\end{aligned}
$$

where $a_{m n}, a_{m n}^{*}, b_{m n}, b_{m n}^{*}$ are Grassmann variables. The disorder parameters $y_{m n}^{2}=0,1$ are still free parameters in the above integral, while Ising degrees being already eliminated. In turn, integrating out a part of fermionic variables from (5) , namely the variables $a_{m n}, b_{m n}$, we obtain the reduced integral for $Q$ in terms of $a_{m n}^{*}, b_{m n}^{*}$ [14]. Changing notation, $a_{m n}^{*}, b_{m n}^{*} \rightarrow c_{m n},-\bar{c}_{m n}$, the integral becomes:

$$
\begin{aligned}
& Q\{y\}=\int \prod_{m n} d \bar{c}_{m n} d c_{m n} y_{m n}^{2} \exp \sum_{m n}\left[y_{m n}^{-2} c_{m n} \bar{c}_{m n}+\right. \\
& \left.+\left(c_{m n}+\bar{c}_{m n}\right)\left(t_{1} c_{m-1 n}-t_{2} \bar{c}_{m n-1}\right)-y_{m n}^{2} t_{1} t_{2} c_{m-1 n} \bar{c}_{m n-1}\right],
\end{aligned}
$$

where $c_{m n}, \bar{c}_{m n}$ are again Grassmann variables, and we assume: $y_{m n}^{2} \exp \left(y_{m n}^{-2} c_{m n} \bar{c}_{m n}\right)=$ $y_{m n}^{2}+c_{m n} \bar{c}_{m n}$, with $y_{m n}^{2}=0,1$. The integrals (5) and (6) are still the exact expressions for $Q\{y\}$ originally defined in (3). Taking $y_{m n}=1$ at all sites, we obtain the 2DIM integral for the pure case:

$$
\begin{aligned}
& Q\{1\}=\int \prod_{m n} d \bar{c}_{m n} d c_{m n} \exp \sum_{m n}\left[c_{m n} \bar{c}_{m n}+\right. \\
& \left.+\left(c_{m n}+\bar{c}_{m n}\right)\left(t_{1} c_{m-1 n}-t_{2} \bar{c}_{m n-1}\right)-t_{1} t_{2} c_{m-1 n} \bar{c}_{m n-1}\right] .
\end{aligned}
$$

In particular, the evaluation of the integral (7) by transformation to the momentum space results the Onsager's expression for $Z$ and $\ln Z$ of the standard rectangular lattice [14]. The advantage of the reduced representation with two variables per site like (6) and (7) is also that it explicitly illuminates the Majorana-Dirac structures of 2DIM already at the lattice level. This can be most easily seen in the pure case. 3

2 Let us remember that Grassmann variables (nonquantum fermionic fields) are the purely anticommuting fermionic symbols. Given a set of Grassmann variables $a_{1}, a_{2}, \ldots, a_{N}$, we have $a_{i} a_{j}+a_{j} a_{i}=0$, and $a_{j}^{2}=0$. The rules of integration over Grassmann variables (fermionic path integral) were originally introduced by F.A. Berezin in QFT context [10. The elementary rules of integration for one variable are [10]: $\int d a_{j} \cdot a_{j}=1, \int d a_{j} \cdot 1=0$. In a multidimensional integral, the differential symbols $d a_{1}, d a_{2}, \ldots, d a_{N}$ are again anticommuting with each other and with the variables. Gaussian fermionic integrals are in general related to the determinants and Pfaffians. In the field-theoretical language, the fermionic form in the exponential under the integral is typically called action. For more comments about Gaussian fermionic integrals in a related context also see [5, 6, 9, 10, 14, 36.

3 In the pure case, the lattice Majorana like action for 2DIM readily follows from (7) by substitution 
To prepare the $N$-replicated integral (마), with same set of $y_{m n}$ in each copy, it is suitable to multiply first (6) by factor $\delta\left(y_{m n}-1\right)+\delta\left(y_{m n}-0\right)=1$, which realizes the decomposition over the states $y_{m n}=0,1$. We assume that $y_{m n}=1$ is realized with probability $p$, and $y_{m n}=0$ is realized with probability $1-p$. The averaging of the $N$ replicated integral (6) over the disorder within the $N$-replica scheme finally results the theory with interaction presented by the integral [14]:

$$
\begin{aligned}
& {\left[Q^{N}\{y\}\right]_{a v}=\int \prod_{m n} \prod_{\alpha=1}^{N} d \bar{c}_{m n}^{(\alpha)} d c_{m n}^{(\alpha)} \prod_{m n}\left[p \prod_{\alpha=1}^{N} \mathrm{e}^{S_{m n}^{(\alpha)}}+(1-p) \prod_{\alpha=1}^{N} c_{m n}^{(\alpha)} \bar{c}_{m n}^{(\alpha)}\right]} \\
& =p^{L^{2}} \int \prod_{m n} \prod_{\alpha=1}^{N} d \bar{c}_{m n}^{(\alpha)} d c_{m n}^{(\alpha)} \exp \sum_{m n}\left[\sum_{\alpha=1}^{N} S_{m n}^{(\alpha)}+\frac{1-p}{p} \prod_{\alpha=1}^{N} c_{m n}^{(\alpha)} \bar{c}_{m n}^{(\alpha)} \mathrm{e}^{-S_{m n}^{(\alpha)}}\right]
\end{aligned}
$$

where $S_{m n}^{(\alpha)}$ is the replicated Gaussian action from (6) for the pure case, see (17). The effect of dilution is introduced here through the second non-Gaussian term, with bar coupling constant $g_{0} \propto \frac{1-p}{p}$. The continuum-limit field theory for weak site dilution (RS 2DIM) that follows from the exact lattice integral (8) is commented in more detail in [14]. This corresponds to the low-momenta sector of the exact lattice theory associated with (6) and (8). To extract the effective low-momenta (long-wave) effective action, one has to distinguish explicitly the higher and low-momentum lattice fermionic modes in the exact lattice action (8). Integrating out the higher-momentum modes in the first order of perturbation theory then results the $N$-colored Gross-Neveu model $(N \rightarrow 0)$ with action [14]:

$$
\begin{aligned}
& S_{\mathrm{G}-\mathrm{N}}=\int d^{2} x\left\{\sum _ { \alpha = 1 } ^ { N } \left[m_{N} \psi_{1}^{(\alpha)} \psi_{2}^{(\alpha)}+\frac{1}{2} \psi_{1}^{(\alpha)}\left(\partial_{1}+i \partial_{2}\right) \psi_{1}^{(\alpha)}\right.\right. \\
& \left.\left.+\frac{1}{2} \psi_{2}^{(\alpha)}\left(-\partial_{1}+i \partial_{2}\right) \psi_{2}^{(\alpha)}\right]+g_{N}\left[\sum_{\alpha=1}^{N} \psi_{1}^{(\alpha)} \psi_{2}^{(\alpha)}\right]^{2}\right\} \\
& m_{N}=\frac{1-t_{1}-t_{2}-t_{1} t_{2}}{\sqrt{2\left(t_{1} t_{2}\right)_{c}}}+\langle A\rangle^{N} \frac{1-p}{p} \frac{\langle B\rangle}{\langle A\rangle} \frac{1}{\sqrt{2\left(t_{1} t_{2}\right)_{c}}} \\
& g_{N}=\langle A\rangle^{N} \frac{1-p}{p} \frac{\langle B\rangle^{2}}{\langle A\rangle^{2}} \frac{1}{4\left(t_{1} t_{2}\right)_{c}}
\end{aligned}
$$

where $\psi_{1}, \psi_{2}$ are the anticommuting Majorana components, $m_{N}$ and $g_{N}$ are the effective mass and charge, respectively. The parameters $\langle A\rangle$ and $\langle B\rangle$ are some lattice fermionic averages (definite numbers) explicitly calculated in [14]. Since the replica limit $N \rightarrow 0$ is assumed at final stages, one can put $N=0$ and $\langle A\rangle^{N}=1$ in mass and charge already in (9). The Gaussian part in (9) is the replicated Majorana action, corresponding to the pure case, with the mass term modified by disorder. The condition of zero mass will give the

$c_{m-1 n} \rightarrow c_{m n}-\partial_{m} c_{m n}, \bar{c}_{m n} \rightarrow \bar{c}_{m n}-\partial_{n} \bar{c}_{m n}$, where $\partial_{m}, \partial_{n}$ are lattice derivatives (momenta). This results the Majorana like action $S=\bar{m} c_{m n} \bar{c}_{m n}+\ldots$ with mass term and the kinetic part [14]. Evidently, the mass parameter will be $\bar{m}=1-t_{1}-t_{2}-t_{1} t_{2}$. The condition $\bar{m}=0$ defines the critical point of $2 \mathrm{DIM}$ in pure case [14. This condition $\bar{m}=0$ may be rewritten as well in the form: $\sinh \left(2 b_{1}\right) \sinh \left(2 b_{2}\right)=1$. The disordered phase corresponds to positive mass, while the ordered phase corresponds to negative mass. 
coexisting curve $\left(T_{c}, p\right)$ for quenched site dilution, which is exact for small concentration of vacancies, as $p \rightarrow 1$. The analysis of that curve at strong and moderated dilution, that is coded in the exact integrals like (5), (8) , has not yet been performed in detail. This will probably claim for the advanced methods of approximation like lattice RG or application of variational approaches like Hartree-Fock-Bogoliubov.

The effective continuum-limit $N=0$ Gross-Neveu model similar to (9), but with another $m_{N}$ and $g_{N}$, has been originally derived and analyzed by DD-SSL as an effective theory near $T_{c}$ for weak bond dilution [11, 12, 13, 24, 28]. The DD-SSL predictions for weak bond dilution, based on the renormalization group (RG) analysis of their effective $N=0$ Gross-Neveu model in the low momentum sector, with taking also into account some fine symmetry effects related to the Kramers-Wannier duality and the CFT interpretations of 2DIM in the pure case, are the double-logarithmic singularity in the specific heat and the logarithmic corrections to the pure-case power laws in other thermodynamic functions, as $T \rightarrow T_{c}$ [11, 12, 13, 24, 28]. The derivation of the effective action for site dilution in the $N=0$ Gross-Neveu form (9) thus supports the idea of the double-logarithmic singularity in specific heat and only logarithmic corrections to the pure-case power laws in other functions (as in the DD-SSL scheme) also for random-site 2D Ising ferromagnets, for weak quenched dilution [14]. For more details and a recent discussion of the effects of quenched disorder in RB and RS versions of 2DIM along theoretical and experimental (Monte-Carlo) lines also see [19, 22, 23, 24, 25, 26, 27, 28, 29, 30, 31]. In conclusion, we note that the fermionic integrals like (5), (6) and (18) are still the exact lattice expressions for $Z$ (either its reduced version $Q$ ). Respectively, one can try other methods of the averaging as well as other tools of analysis of fermionic theories of random-site 2DIM directly on a lattice, starting from these exact fermionic integrals.

\section{The Blume-Capel model}

The Blume-Capel (BC) model is a classical spin-1 model originally introduced to study phase transitions in specific magnetic materials with a possible admixture of non-magnetic states. This is a model with the tricritical point at the critical line in $\left(T_{c}, \Delta_{0}\right)$ plane [32, 33, 34, 38]. The Blume-Capel model can also be viewed as the annealed site-dilute version of the ordinary Ising model [36]. The hamiltonian is:

$$
H=-\sum_{m=1}^{L} \sum_{n=1}^{L}\left[J_{1} S_{m n} S_{m+1 n}+J_{2} S_{m n} S_{m n+1}\right]+\Delta_{0} \sum_{m=1}^{L} \sum_{n=1}^{L} S_{m n}^{2},
$$

where $S_{m n}=0, \pm 1$ is the BC spin-1 variable associated with the $m n$ lattice site $(m, n=$ $1,2,3, \ldots, L)$. As distinct from the quenched disorder case, the zero-spin or vacancy state of $S_{m n}=0, \pm 1$ is now rather to be considered as a one of the three possible states of spin variable [32, 33, 35, 36]. The hamiltonian modulus in the Gibbs exponential is:

$$
-\beta H=\sum_{m=1}^{L} \sum_{n=1}^{L}\left[K_{1} S_{m n} S_{m+1 n}+K_{2} S_{m n} S_{m n+1}\right]+\Delta \sum_{m=1}^{L} \sum_{n=1}^{L} S_{m n}^{2},
$$

with $K_{1,2}=\beta J_{1,2}$ and $\Delta=-\beta \Delta_{0}$. The decomposition like $S_{m n}=y_{m n} \sigma_{m n}$, that was used in the quenched case, is still possible, but in order to eliminate the Ising degrees by 
transformation of $Z$ into a fermionic integral it is now more suitable to make use rather of the gauge transformation like $S_{m n} \rightarrow \sigma_{m n} S_{m n}$ under the averaging, since the product of cosines (factor $R\{y\}$ ) is to be included into $Z$ anyhow, before averaging. The Boltzmann factors from the Gibbs exponential associated with (11) can be written as (extended) polynomials in variables $S_{m n}=0, \pm 1$. The polynomial interpretation is important for fermionization [36]. The partition function becomes (with $\lambda_{i}=\sinh K_{i}, \lambda_{i}^{\prime}=\cosh K_{i}-$ $1)$ :

$$
\begin{array}{r}
Z=\operatorname{Sp}_{(S)}\left\{\prod _ { m = 1 } ^ { L } \prod _ { n = 1 } ^ { L } e ^ { \Delta S _ { m n } ^ { 2 } } \left[\left(1+\lambda_{1} S_{m n} S_{m+1 n}+\lambda_{1}^{\prime} S_{m n}^{2} S_{m+1 n}^{2}\right)\right.\right. \\
\left.\left.\times\left(1+\lambda_{2} S_{m n} S_{m n+1}+\lambda_{2}^{\prime} S_{m n}^{2} S_{m n+1}^{2}\right)\right]\right\} .
\end{array}
$$

The factorization of local bond weights can again be performed by analogy with the Ising case, but now we have to add the even part of the polynomial into the Gaussian exponential in the measure [36]. For the horizontal weights, with Grassmann variables $a_{m n}, \bar{a}_{m n}$, we write:

$$
\begin{aligned}
& 1+\lambda_{1} S_{m n} S_{m+1 n}+\lambda_{1}^{\prime} S_{m n}^{2} S_{m+1 n}^{2}= \\
& =\int d \bar{a}_{m n} d a_{m n} \exp \left\{\left(1+\lambda_{1}^{\prime} S_{m n}^{2} S_{m+1 n}^{2}\right) a_{m n} \bar{a}_{m n}\right\} \\
& \times\left(1+a_{m n} S_{m n}\right)\left(1+\lambda_{1} \bar{a}_{m n} S_{m+1 n}\right)
\end{aligned}
$$

and similarly we can factorize the vertical bond Boltzmann weights. The factorization (13) makes it possible to pass to the purely fermionic expression for $Z$ in few steps. The final fermionic integral for $Z$ appears in the form [36]:

$$
\begin{aligned}
& Z=\left(2 e^{\Delta} \cosh K_{1} \cosh K_{2}\right)^{L^{2}} \int \prod_{m=1}^{L} \prod_{n=1}^{L} d \bar{a}_{m n} d a_{m n} d \bar{b}_{m n} d b_{m n} \\
& \times \exp \left\{\sum _ { m = 1 } ^ { L } \sum _ { n = 1 } ^ { L } \left[a_{m n} \bar{a}_{m n}+b_{m n} \bar{b}_{m n}+a_{m n} b_{m n}\right.\right. \\
& +\left(t_{1} \bar{a}_{m-1 n}+t_{2} \bar{b}_{m n-1}\right)\left(a_{m n}+b_{m n}\right)+t_{1} t_{2} \bar{a}_{m-1 n} \bar{b}_{m n-1} \\
& +g_{0} a_{m n} \bar{a}_{m n} b_{m n} \bar{b}_{m n} \exp \left(-\gamma_{1} a_{m-1 n} \bar{a}_{m-1 n}-\gamma_{2} b_{m n-1} \bar{b}_{m n-1}\right. \\
& \left.\left.\left.-t_{1} t_{2} \bar{a}_{m-1 n} \bar{b}_{m n-1}\right)\right]\right\}
\end{aligned}
$$

with parameters (where $\left.\Delta=-\beta \Delta_{0}\right)$ :

$$
g_{0}=\frac{e^{-\Delta}}{2 \cosh K_{1} \cosh K_{2}}, \quad \gamma_{i}=1-\frac{1}{\cosh K_{i}}=1-\sqrt{1-t_{i}^{2}} .
$$

The fermionic integral (14) is still the exact expression, even for finite lattices, provided we assume free boundary conditions both for spins and fermions. The exponential in the interaction term can be expanded into a series, which results a finite polynomial in Grassmann variables. For instance, for particular exponential factor we find: $\exp \left(-\gamma_{1} a_{m-1 n} \bar{a}_{m-1 n}\right)=1-\gamma_{1} a_{m-1 n} \bar{a}_{m-1 n}$, and analogously one can expand other factors. 
The B-C model is thus presented in (14) as a fermionic theory with free-fermion (Gaussian) part and a polynomial interaction terms in the action, the highest term in the interaction polynomial is of order 8 in fermions. The overall coupling constant in interaction term is $g_{0} \propto \exp \left\{\beta \Delta_{0}\right\}$, the increasing $g_{0} \propto \exp \left\{\beta \Delta_{0}\right\}$ means increasing dilution. There are also additional parameters $\gamma_{1,2}$ in the interaction polynomial. These parameters come from accounting properly the weights related to factors $\cosh \left(b_{1} S_{m n} S_{m+1 n}\right) \cosh \left(b_{2} S_{m n} S_{m n+1}\right)$ in $Z\{S\}$ for Blume-Capel, there are no analogs of $\gamma_{1,2}$ terms in the quenched case. In fact, these terms with $\gamma_{1,2}$ are responsible for the existence of the tricritical point in the Blume-Capel model. The elimination of the variables like $a_{m n}, b_{m n}$ is now not possible in action (14), at least straightforwardly, as distinct from the quenched case (cf. (6) $-(17)$ ), just because of the presence of the combinations like $a_{m n} a_{m-1}$ and $b_{m n} b_{m n-1}$ in the $\gamma_{1,2}$ terms in the non-Gaussian part of the lattice action (14).

Despite of the non-Gaussian representation for $Z$, it is still possible to extract physical information by taking the continuous limit (low momenta sector) of the BC lattice action like (14) and analyzing it using tools from quantum field theory. The details of constructing the effective two-component fermionic action at low momenta can be seen in [36]. The resulting action includes the Gaussian part, with mass term modified by disorder, and the four-fermion interaction of the form $\left(\psi \bar{\psi} \mid \partial_{x} \psi \partial_{y} \bar{\psi}\right)$. The condition of the zero effective mass already gives the equation for the $\mathrm{BC}$ line of phase transitions (critical line) in the $\left(T_{c}, \Delta_{0}\right)$ plane, while the effect of the interaction is merely to modify the kinetic terms in the action [36]. This also provide grounds to estimate the position of the tricritical point [36]. These effects are shortly commented below.

The critical line is given by the condition of vanishing the mass term in effective BC action: $m_{B C}=1-t_{1}-t_{2}-t_{1} t_{2}+g_{0}=0$, where $g_{0}$ is given in (15). Following [36], we now consider the isotropic lattice case, with $t_{1}=t_{2}=t$ and $K_{1}=K_{2}=K$. The critical line is given by $m_{B C}=1+g_{0}-2 t-t^{2}=0$, with $t=\tanh K$ and $K=\beta J$, where $\beta=1 / T$. In notation with $K=\frac{J}{T} \rightarrow \frac{1}{T}$ and $\frac{\Delta_{0}}{J} \rightarrow \Delta_{0}$, the criticality condition becomes:

$$
\tanh ^{2}\left(\frac{1}{T}\right)+2 \tanh \left(\frac{1}{T}\right)-1=\frac{e^{\frac{\Delta_{0}}{T}}}{2 \cosh ^{2}\left(\frac{1}{T}\right)}
$$

which may be written as well in the form:

$$
\sinh \left(\frac{2}{T}\right)=1+\frac{1}{2} \exp \left(\frac{\Delta_{0}}{T}\right)
$$

which in turn admits the explicit solution for $\Delta_{0}$ as function of $T=T_{c}$ in the form:

$$
\Delta_{0}=T \ln \left[2 \sinh \left(\frac{2}{T}\right)-2\right]
$$

The inverse dependence for $T_{c}$ as function of $\Delta_{0}$ can be evaluated numerically by solving any of the above equations, which are all equivalent to the condition of the zero mass in the effective continuum-limit theory that follow from (14). This results the critical line for the $\mathrm{BC}$ model shown in Fig. 1 in [36. The critical line is started with maximal $T_{c}$ at the left end at $\Delta_{0}=-\infty$, which corresponds to the pure case $\left(g_{0}=0\right)$, and goes lower as dilution increases, with increasing $\Delta_{0}$ and $g_{0} \propto \exp \frac{\Delta_{0}-2}{T}$. The critical line finally terminates at 


\begin{tabular}{c|ccc}
\hline $\begin{array}{c}\text { Chemical potential } \\
\Delta_{0}\end{array}$ & Ref. [33] & Temperature $T_{c}\left(\Delta_{0}\right)$ & Ref. [35] \\
\hline & & & Eqs. (16) $-(18)$ \\
\hline .0 & 1.695 & $1.714(2)$ & 1.6740 \\
0.5 & 1.567 & $1.584(1)$ & 1.5427 \\
1.0 & 1.398 & $1.413(1)$ & 1.3695 \\
1.5 & 1.150 & $1.155(1)$ & 1.1162 \\
1.87 & 0.800 & $0.800(3)$ & 0.7712 \\
1.95 & 0.650 & $0.651(2)$ & 0.6135 \\
1.962 & 0.620 & $0.619(1)$ & 0.5776 \\
1.969 & 0.600 & $0.596(5)$ & 0.5531 \\
\hline
\end{tabular}

Table 1: Numerical values of the critical points $\left(T_{c}\left(\Delta_{0}\right), \Delta_{0}\right)$ in the Blume-Capel model: comparison of the results of Monte-Carlo simulations and the equations (16)-(18). Note that small variation of $\Delta_{0}$ causes more significant changes in $T_{c}\left(\Delta_{0}\right)$ in the region near $\Delta_{0}=2$, as it is to be expected from (16)-(18).

$\Delta_{0}=2$ at zero temperature. There is no ordered phase at stronger dilution, as it also can be deduced from (16)-(18). The theoretical critical line is compared with the results of the recent Monte-Carlo simulations for B-C model, see Fig. 1 in [36]. The agreement is found to be very good (typically within 1\% accuracy) over the whole temperature range [36]. The available numerical data for $\left(T_{c}, \Delta_{0}\right)$ are also presented (in part) in Table 1.

The position of the tricritical point at the $\left(T_{c}, \Delta_{0}\right)$ line can as well be estimated from the condition of vanishing the kinetic (stiffness) coefficient in the effective B-C action associated with (14) 36]. The Hartree-Fock-Bogoliubov method has been applied to decouple the four-fermion interaction term in the effective action to extract the corrections to the kinetic part [36]. The singular point where the kinetic coefficient vanishes was found at $\left(T_{t}^{*}, \Delta_{0, t}^{*}\right) \simeq(0.42158,1.9926)$, in a reasonably good agreement with the results of Monte-Carlo simulations for the position of tricritical point: $\left(T_{t}, \Delta_{0, t}\right) \simeq(0.610,1.9655)$ [33], and $\left(T_{t}, \Delta_{0, t}\right) \simeq(0.609(3), 1.966(2))$ 35]. It is in general important that the B$\mathrm{C}$ fermionic integral with a non-Gaussian action (14) finally predicts the existence of a tricritical point at the $\mathrm{B}-\mathrm{C}$ critical line at strong dilution, somewhere close to the termination point of that line at $\Delta_{0}=2$. The method of constructing the critical line from the condition of zero mass in fermionic integral for $Z$ has been recently extended by Fortin and Clusel [37] to the general set of the spin- $S$ Ising models $(S=1 / 2,1,3 / 2,2,5 / 2, \ldots)$ [37]. The standard 2DIM and the Blume-Capel models are the first two representatives in this set. The agreement of the theoretical predictions for the critical line with the available Monte-Carlo data for the spin- $S$ models was again found to be very good, even despite of highly complicated polynomial structures, with many fermions, arising for the higher spin- $S$ Ising models in the kinetic part of the action [37]. These features may be probably understood as an evidence for the well expressed clusterization processes at low temperatures in such models, including the generic case of the spin-1 Blume-Capel model. 


\section{Conclusions}

The integrals with anticommuting (Grassmann) variables provide effective tools to analyze pure and disordered Ising like spin models in two dimensions. The Ising spin glasses, geometry disordered lattices, regularly diluted models, also can be analyzed along these lines. In a more general context, it may be noted that there are as well few other important physical problems with spins and fermions in two dimensions. The most prominent are the quantum Hall effect and the high- $T_{c}$ superconductivity in oxide cuprates and related substances. It is interesting that the fine tuning in spin-fermion correspondence as well as the effects of disorder seemingly play the role in both cases. It is therefore important to understand better the ordering phenomena in terms of fermions in such systems.

\section{References}

[1] L. Onsager, In: Critical Phenomena in Alloys, Magnets and Superconductors, Ed. by R.E. Mills, E. Ascher, R.I. Jaffe (McGraw-Hill, New York, 1971), p. XIX-XXIV; p. $3-12$.

[2] T.D. Schultz, D.C. Mattis, E.H. Lieb, Rev. Mod. Phys. 36, 856 (1964).

[3] E.W. Montroll, R.B. Potts, J.C. Ward, J. Math. Phys.4, 308 (1963).

[4] C.A. Hurst and H.S. Green, J. Chem. Phys. 33, 1059 (1960).

[5] F.A. Berezin, Russ. Math. Surveys, 24, No. 3, 1 (1969).

[6] S. Samuel, J. Math. Phys. 21, 2806 (1980).

[7] C. Itzykson, Nucl. Phys. B 210, 448 (1982).

[8] V.N. Plechko, Sov. Phys. Doklady, 30, 271 (1985).

[9] V.N. Plechko, Theor. Math. Phys. 64, 748 (1985).

[10] F.A. Berezin, The Method of Second Quantization (Academic Press, New York, 1966).

[11] V.S. Dotsenko and V.S. Dotsenko, Adv. Phys. 32, 129 (1983).

[12] B.N. Shalaev, Phys. Rep. 237, 129 (1994).

[13] G. Jug and B.N. Shalaev, Phys. Rev. B 54, 3442 (1996).

[14] V.N. Plechko, Phys. Lett. A 239, 289 (1998).

[15] A. Roder, J. Adler, and W. Janke, Phys. Rev. Lett. 80, 4697 (1998).

[16] G. Mazzeo and R. Kühn, Phys. Rev. E 60, 3823 (1999).

[17] J.-K. Kim, Phys. Rev. B 61, 1246 (2000).

[18] K. Ziegler, Nucl. Phys. B 344, 499 (1990).

[19] L.N. Shchur and O.A. Vasilyev, Phys. Rev. E 65, 016107 (2001).

[20] V.N. Plechko and I.K. Sobolev, Phys. Lett. A, 157, 335 (1991).

[21] V.N. Plechko and I.K. Sobolev, Physica A, 197, 323 (1993). 
[22] W. Selke, L.N. Shchur, and O.A. Vasilyev, Physica A 259, 388 (1998).

[23] H.J. Luo, L. Schülke, and B. Zheng, Phys. Rev. E 64, 036123 (2001).

[24] M. Picco, A. Honecker, and P. Pujol, J. Stat. Mech.: Theory Exp. (2006) P09006.

[25] P.H.L. Martins and J.A. Plascak, Phys. Rev. E 76, 012102 (2007).

[26] M. Hasenbusch, F.P. Toldin, A. Pelissetto, and E. Vicari, Phys. Rev. B 78, 011110 (2008).

[27] R. Kenna and J.J. Ruiz-Lorenzo, Phys. Rev. E 78, 031134 (2008).

[28] A. Gordillo-Guerrero, R. Kenna, and J.J. Ruiz-Lorenzo, AIP Conf. Proc. 1198, p.4254, 2009. Also see: arXiv:0909.3774.

[29] N.G. Fytas, A. Malakis, and I.A. Hadjiagapiou, J. Stat. Mech.: Theory Exp., P11009 (2008).

[30] A. Malakis, A. Nihat Berker, I.A. Hadjiagapiou, N.G. Fytas, Phys. Rev. E, 79, (2009).

[31] X.T. Wu and J.Y. Zhao, Phys. Rev. B, 80 (2009) 104402.

[32] M. Blume, V.J. Emery and R.B. Griffiths, Phys. Rev. A, 4, 1071 (1971).

[33] P.D. Beale, Phys. Rev. B, 33, 1717 (1986).

[34] Y.Deng, W.Guo, and H.W.Blöte, Phys. Rev. E 72, 016101 (2005).

[35] C.J. Silva, A.A. Caparica, and J.A. Plascak, Phys. Rev. E, 73, 036702 (2006).

[36] M. Clusel, J.-Y. Fortin, and V.N. Plechko, J. Phys. A: Math. Theor., 41, 405004 (2008).

[37] J.-Y. Fortin and M. Clusel, Phys. Rev. B, 78, 172402 (2008).

[38] I.D. Lawrie and S. Sarbach, in Phase Transitions and Critical Phenomena, edited by C. Domb and J.L. Lebowitz (Academic Press, London, 1984), Vol. 9, p. 1. 